\title{
Rilievo magnetometrico del giacimento di Fornovolasco
}

\author{
M. BERNABINI
}

Ricevuto il 5 Luglio 1965

Riassunto. - Sono descritte le operazioni di campagna ed i metodi usati nell'interpretazione di un rilievo magnetometrico eseguito nella zona del giacimento di pirite e magnetite di Fornovolasco (Lucca), sito sulle pendici della Pania Secca (Alpi Apuane). Mediante le determinazioni di $\Delta I I$, $\Delta Z$ e $\Delta D$ è stato possibile ricavare in ogni stazione il vettore anomalo totale. L'interpretazione è stata basata sull'uso di modelli costituiti da elettrocalamite prismatiche. I risultati dell'interpretazione sono discussi sulla base delle conoscenze geominerarie sul giacimento.

Summary. - The A. deals with the field operations and the methods used in the interpretation of a magnetometric survey on the "Fornovolasco" pyrite and magnetite ore body. The anomalous $\Delta H, \Delta Z$ and $\Delta D$ were measured; therefore it was possible to calculate the total anomalous vector in every station. The $A$. used model s, consisting in prismatic electro-magnets, to interpretate the data. The results are discnssed on the bas is of geology of the deposit.

\section{1. - Premesse.}

Sul versante sudorientale della Pania Secca nel gruppo delle Alpi Apuane si rinvengono alcune manifestazioni di minerali di ferro. Ia più importante è conosciuta con il nome di Fornovolaseo, dalla piccola frazione del comune di Vergemoli (Iucea) che dista dalla manifestazione circa un chilometro in linea d'aria.

Dopo alcune coltivazioni effettuate fin dal Medioevo [3-4] ricerche saltuarie, più o meno sistematiche, furono compiute da parte di varie Società concessionarie a partire dal 1906 fino ai nostri giomi.

L'Istituto di Geofisica Mineraria dell'Università di Roma, ha eseguito nel 1957 un rilievo magnetometrico la cui interpretazione è stata curata dallo scrivente che ha effettuato anche le operazioni di campagna. 
Scopi del rilievo erano la deteminazione dellestensione della mineralizzazione a magnetite già riconosciuta con le indagini dirette a la individuazione di altre masse eventualmente presenti.

Nell'esecuzione delle misure a nell'interpretazione sono stati seguiti alcuni criteri ed accorgimenti, che si ritiene possano esser utili per rilievi di tipo analogo. Per tale ragione con la presente nota, si vogliono portare a conoscenza le operazioni di campagna e il metodo utilizzato nell'interpretazione del rilievo magnetometrico; si ringraziano i dirigenti della societa IMSA per il permesso alla pubblicazione dei risultati.

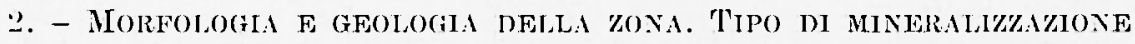 PRESENTE.}

Ial zona interessata dal rilievo magnetometrico è costituita essenzialmente da uno sperone (Fig. 1) che si protende verso SE dalla massa della Pania Secea. I versanti si presentano acclivi; sul versante verso $\mathbf{S E}$, in corrispondenza dei principali affioramenti di minerale, il pendio è dirupato a quasi verticale per un'altezza di cirea $20-30 \mathrm{~m}$.

In base alle informazioni raccolte e alle notizie riportate sulla letteratura [2-3-4-5] il giacimento è costituito da diverse masse di piccole dimensioni (dell'ordine di alcune centinaia di me. al massimo) grossolanamente lenticolari, costituite principalmente da magnetite e da pirite, situate (vedi Fig. 1) presso il contatto tra gli "Scisti carnici " [2] (scisti sericeitici, microanageniti ecce) e soprastanti rocee calcareo-dolomitiche (" arernoso ") [?] $\left.{ }^{*}\right)$.

La superlicie di contatto mediamente avrebbe direzione prossima NNE-SSO a debole immersione verso IVNW. Ia mineralizzazione interesserebbe sia gli scisti di letto sia i caleari di tetto $\left[^{2}\right]$.

Magnetite e pirite costituirebbero in genere concentrazioni abbastanza distinte con scarse porzioni di minerale misto.

Le antiche coltivazioni effettuate e le ricerche eseguite dalle Societa Concessionarie avevano messo in vista una fascia mineralizzata a magne-

$\left(^{*}\right)$ La carta geologica della Fig. 1 è ripresa dalla recente nota di NARDI $\left[{ }^{2}\right]$ Le formazioni citate sono attribuite al Trias e tettonicamente farehbero parte del "Complesso della Pania Secea - Pania della Crocen. Le mineralizzazioni interesserebhero la breccia di frizione tra le due formazioni sudclette. Da studi in corso sul giacinento sembra che la situazione geologica locale sia in effetti, nel dettaglio, più complessa di quanto appaia nella allegata cartina.

Questa tuttavia è sufficientemente approssimata per inquadrare il problemil geofisico. 


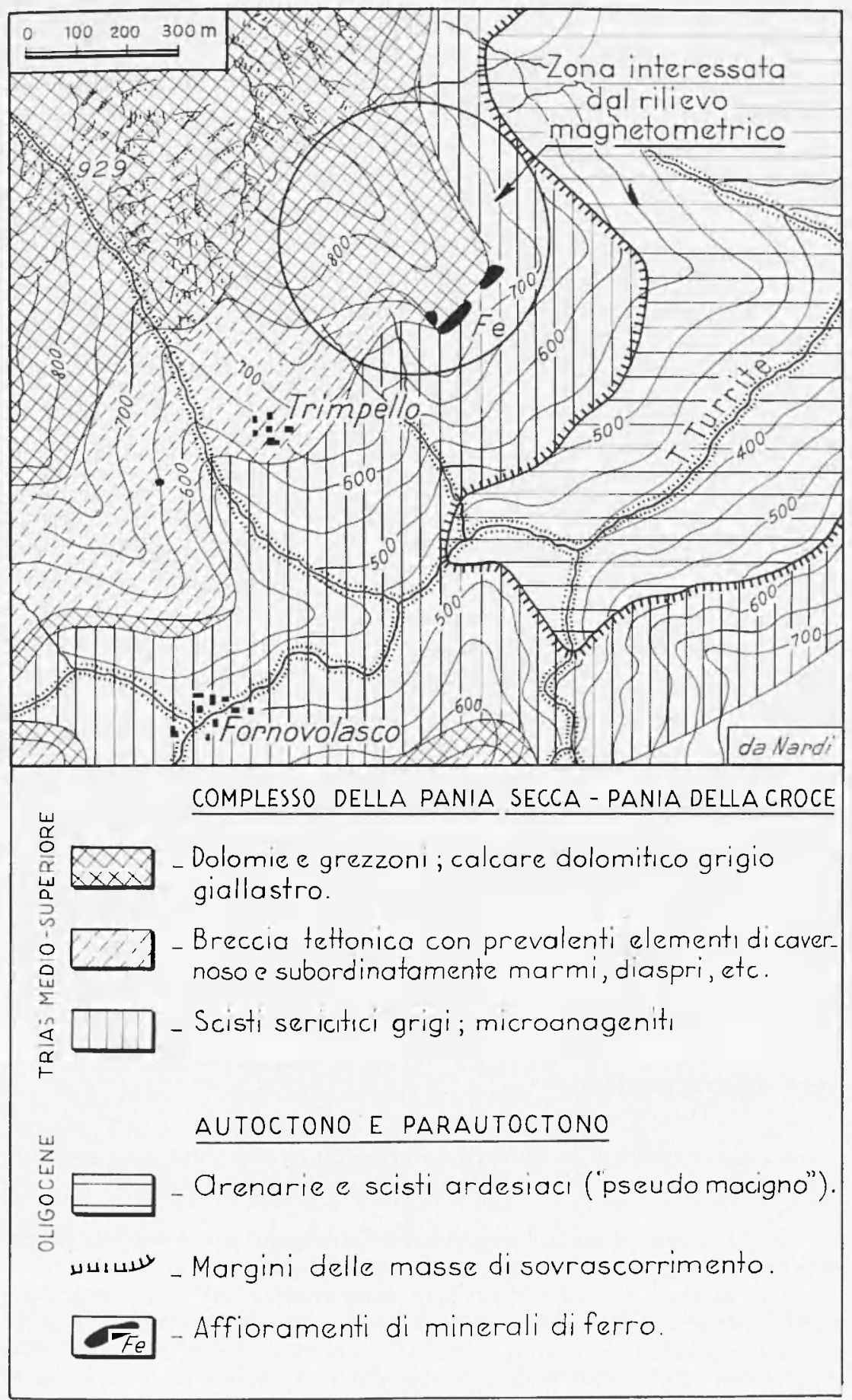

Fig. I - (arta geologiea della zona (da Nardi $\left[{ }^{2}\right]$ ). 
tite e pirite. A seguito dei lavori minerari, in prossimità degli affioramenti e degli sbocchi a giorno delle gallerie si rinvengono tuttora sparsi mucchi e blocchi isolati di minerale.

\section{3. - Misure Mignetometriche esegtitte.}

Preliminarmente furono effettuate determinazioni della componente anomala $(\Delta Z)$ del campo magnetico terrestre su due allineamenti sviluppantisi con direzione circa NW-SE, cioè grosso modo parallelamente alla cresta dello sperone. Già una prima interpretazione di queste misure fece sospettare la presenza di masse di minerale in una zona ancora inesplorata. Si decise perciò la prosecuzione del rilievo con misure sia delle componenti anomale verticale ed orizzontale che della declinazione anomala. Ciò avrebbe infatti permesso la ricostruzione del vettore anomalo totale e quindi un'interpretazione pì̀ sicura dei risultati delle misure.

Per la misura delle componenti anomale verticale ed orizzontale è stata utilizzata una bilancia magnetica di Sohmidt tipo GF-6 della Askania Werke di Berlino; la sensibilita degli agrhi era di circa 25y (*).

Con un'altra bilancia dello stesso tipo (ma con aghi di circa $10 \gamma$ per parte), si effettuava la determinazione della variazione diurna della stessa componente del campo magnetico terrestre che si stava misurando.

Per la misura della declinazione magnetica si è utilizzato un tacheometro Wildt To, il cui cerchio azimutale può autorientarsi sul Nord magnetico; l'approssimazione di lettura degli angoli è del primo centesimale $\left(^{* *}\right)$. Si sono misurate le declinazioni del campo magnetico rispetto alle direzioni degli allineamenti sui quali erano state ubicate le stazioni. Poiché tali allineamenti elano stati collegati tra loro da poligonali topografiche, si sono riferite tutte le declinazioni ad un'unica direzione scelta arbitrariamente.

$\left(^{*}\right)$ Il controllo della sensibilità degli aghi veniva effettuata con una bobina di Helmholtz all'inizio e alla fine di ogni giomata di lavoro di campagna.

(**) I'er controllare la precisione dello strumento per la determinazione delle declinazioni relative, sono state ripetute integralmente numerose misure in alcune stazioni. Gli errori medi nelle varie stazioni non hanno superato i 2 primi centesimali. Non sono state considerate le declinazioni assolute rispetto al Nord geografico, in quanto l'eventuale non coincidenza dello zero della graduazione con il Nord magnetico (errore sistematico) non influenza la determinazione delle declinazioni anomale. 
Il controllo dell'andamento diurno della declinazione è stato eftettuato rioccupando, ad intervalli costanti di tempo, una stessa stazione; contemporaneamente, alla stazione base, si registravano le variazioni della componente orizzontale, per determinare l'eventuale verificarsi di brusche variazioni nel campo magnetico terrestre.

\section{4. - CoRREZioni ED ELABorazioni DEgLt Elementi Misurati.}

Alle misure delle componenti orizzontale e verticale sono state apportate le correzioni per $\mathrm{i}$ magnetini ausiliari, per la variazione diurna e per la stazione base; per la componente orizzontale si è effettuata anche la correzione per l'influenza delle variazioni da punto a punto della componente verticale [1].

Per determinare $\mathrm{i}$ valori anomali delle componenti nella stazione base, si sono prese in considerazione numerose stazioni situate all'intorno della zona presumibilmente mineralizzata entro un raggio di circa $400 \mathrm{~m}$.

I valori di $\Delta Z$ e $\Delta H$ di tali stazioni rispetto alla stazione base sono risultati compresi entro $\pm 10 \gamma$ intorno ai valori medi $\left({ }^{*}\right)$.

Le anomalie delle due componenti determinate nelle varie stazioni sono state corrette dei valori medi così ottenuti, ricavando quindi le anomalie rispetto ai valori medi "regionali " (**).

Alle misure di declinazione non sono state apportate correzioni per l'andamento diurno, dato che, nei pochi casi in cui erano riscontrate variazioni apprezzabili (oltre i 2 primi centesimali), le misure sono state ripetute.

Come valore medio "regionale " della declinazione, è stata assunta la media delle misure effettuate nei punti più lontani dalla zona presumibilmente mineralizzata; punti nei quali si erano ottenuti valori praticamente nulli sia di $\Delta Z$ che di $\Delta H$.

Si sono avuti così a disposizione, per ogni stazione, i valori anomali, rispetto alle medie "regionali ", di $\Delta Z, \Delta H$ e $\Delta D$.

I valori di $\Delta H$ e $\Delta D$ sono stati utilizzati per la determinazione, in grandezza e direzione, della componente orizzontale anomala totale $\left(\Delta H_{i}\right)$.

$\left(^{*}\right)$ Si sono riscontrate sistematicamente differenze molto forti(ancho oltre i $50 \%$ ) su alcune piazzole di carbonaie.

(**) Data la ristrettezza della zona non si considerano le variazioni dei valori regionali con le coordinate geografiche. 
Il valore di $A H$ è infatti la differenza tra il modulo del vettore componente orizzontale in un punto (cioe del vettore somma della componente orizzontale "regionale " $H_{r}$ e rella componente orizzontale totale anomala $\left.A \bar{H}_{l}\right)$ e il modulo della componente orizzontale regionale $\bar{H}_{r}$ e l'anomalia di declinazione $A D$ i l'angolo formato dai due rettori. Si possono quincli, con sempliei consirlerazioni geometriche, ricavare, della romponente orizzontale anomala totale $\Delta \bar{H}_{t}$, il modulo, lorientamento a rispetto alla dierzione di $\bar{H}_{r}$ e le due componenti rispetto alla dierzione di $H_{r}\left(\Delta H_{p}\right)$ e rispetto alla direzione normale $\left(\Delta H_{n}\right)$.

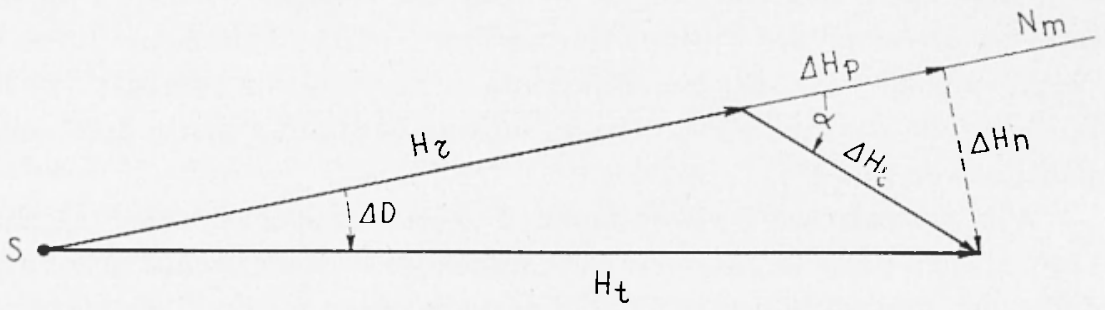

Fig. :

Dallat Fig. 2, considemando come rerso position deggli angoli quello omario, si ha:

$$
\begin{aligned}
& \left|H_{\imath}\right|=\left(\Delta H^{2}+4 H_{r}\left(H_{r}+\Delta H\right) \cdot \operatorname{sen}^{2} \frac{\Delta I}{2}\right)^{1 / 2} \\
& A H_{p} \quad-\quad 1 H \cos 1 D-2 H_{r} \operatorname{sen}^{2} \frac{\Delta I D}{2} \\
& \left.1 H_{n}=\left(H_{r}+\lambda H\right) \operatorname{sen} . A T\right) \\
& \text { lyue }=\frac{\left(H_{r}+\Delta H\right) \operatorname{sen} 1 D}{.1 H \cos \Delta D-2 H_{r} \operatorname{sen}^{2} \frac{A D}{2}} \text {. }
\end{aligned}
$$

Nel caso in esame per $\Delta H$ minori di $200 \gamma$ a per $.1 D$ minori di $0,5^{g}$, in luogo della [1] si possono considerare le esporessioni semplificate:

$$
\begin{aligned}
& |.| H_{\iota}|-| 1 H^{-}+\left.\left(H_{r} \cdot 1 D \text { ale } 1^{g}\right)^{2}\right|^{1 / 2} \\
& 1 H_{p}=\Delta H \\
& 1 H_{n}=H_{r} \Delta D \text { arc } 1^{\prime} \\
& \operatorname{tgre}=\frac{H_{r} \Delta D \operatorname{sic}^{g}}{1 H}
\end{aligned}
$$

(con I) aspressi in grarli contesimali. 
Per la determinazione di $H_{r}$ si è utilizzato il valore fornito dalla casa costruttrice per la posizione orizzontale dell'ago. Tale valore è stato controllato con alcune stazioni effettuate nei pressi di osservatori magnetici in eui era noto il valore assoluto della componente orizzontale. Le differenze rispetto al valore ricavabile in base alla tamatura dell'ago effettuata dalla Casa sono risultate inferiori a $100 \gamma$.

Anche assumendo un errore di $\pm 200 \%$ nella determinazione di $H_{r}$ nella zona di Forno Volaseo rispetto al valore vero, exli errori in $1 H_{\text {, }}$ ed in a sono risultati trascumbili ai fini del pilievo (*).

Sulla base quindi del valore assunto di $H_{r}(23.200 \gamma)$ si sono calcolate per ciascuma stazione i valori di $\Delta H$, ed $a$.

Nella Fig. 3 le componenti orizzontali anomale sono riportate come rettori orientati e le componenti verticali anomale $(\Delta Z)$ ron numeli (**). Nella stessa figura sono rappresentate anche le curve di livello topografiche e le isoanomale di $\triangle Z$.

\section{5. - INTERPRETAZIONE DEI VALORI MISURATI.}

Da una prima analisi della distribuzione dei valori anomali riportati nella Fig. 3, si può notare un generale andamento abbastanza regolare dej ralori di $\triangle Z$ intorno a due zone di massimo, indicate con le lettere $A$ a $B$ nella stessa Figura 3.

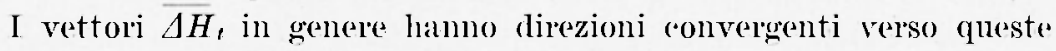
due zone $(A$ a $B$ ) di massimo di $A Z$.

Tali zone sono separate da una fascia di minimo di $\Delta Z$ in comispondenza della quale i vettori $\overline{\Delta H}$, presentano, da punto a punto, variazioni forti e spesso irregolari nel loro orientamento.

(*) Gli errori sono stati calcolati con la formula della propagazione degli errori per funzioni di quantiti osservate:

$$
H_{A}=\sqrt{\left(\frac{\partial A}{\partial x}\right)^{2} m^{2} x+\left(\frac{\partial A}{\partial y}\right)^{2} m^{2} y+\cdots}
$$

Ad es. nel caso in esame per $\Delta I I-1000$ y e $D=3^{q}$, gli errori in $\Delta H_{q}$ ed in u. dovuti ad $u n$ errore di $200 y$ in $H_{r}$, sono rispettivamente di $8 y$ e $12^{c}$.

$\left(^{* *}\right)$ In aleune stazioni situate ai bordi della zona rilevata non furono eseguite le misure della declinazione. Poiché le anomalie di $d z$ e $1 / /$ risultarono in menere inferiori o di poeo superiori ai $200 \%$, in base alle (3) si sono riportate in Fig. 3 le sole componenti nella direzione di $I_{r}\left(. H_{p}\right)$. 
La zona di massimo contrassegnata dalla lettera $A$ è situata in corrispondenza delle masse mineralizzate già individuate. Nelle zone di affioramento della mineralizzazione, ove, come si è detto, sboccano numerose gallerie, si riscontrano valori anomali molti alti (superiori a $1000 \gamma$ fino ad un valore di $11.600 \gamma$ ). In prossimità degli imbocchi delle gallerie le anomalie divengono irregolari, in valore ed orientamento. Ciò è senza dubbio dovuto alla presenza di blocchi e frammenti di minerale che si trovano sparsi in tale zona (*). Per tale ragione i valori che scartavano nettamente rispetto ad un andamento generale non sono stati considerati nell'interpretazione.

Anche escludendo tali valori, la configurazione delle anomalie risulta non molto semplice. Tue due zone di massimo $A$ e $B$ non sono simili fra loro e mostrano alcuni andamenti irregolari sia nel valore di $\Delta Z$, sia nel valore e nell'orientamento di $\Delta \bar{H}_{\imath}$.

I'andamento delle anomalie è senza dubbio condizionato dalla conformazione topografica della zona, cui possono essere dovute, in tutto o in parte, alcune delle irregolarità che si riscontrano nelle anomalie stesse.

Un primo tentativo di interpretazione è stato eflettuato schematizzando le masse magnetiche con un numero limitato di dipoli (da 2 a 5 ).

Il calcolo teorico delle anomalie è risultato laborioso, data la topografia della zona, e non ha portato a risultati soddisfacenti. Per una ricostruzione anche di prima approssimazione si verifico che sarebbe stato necessario considerare non meno di 10-15 dipoli.

Per rendere l'interpretazione più spedita, si è ricorso all'analisi su modelli [1].

Si sono materializzati i dipoli con elettrocalamite $\left[^{1}\right]\left(^{* *}\right)$ costituite dir un nucleo prismatico abbracciato da un avvolgimento in rame.

Si avevano a disposizione elettrocalamite di diverse dimensioni assiali e con diversi numeri di spire $(* * *)$. Si aveva inoltre la possibilità di variare l'intensità della corrente continua che veniva fatta circolare nelle

(*) Per tale ragione non si sono effettuate stazioni sui versanti a quote inferiori a quelle degli imbocchi delle gallerie. Anche in prossimità di queste, alcune stazioni ricalenti nelle vicinanze di muchi di minerale furono scartate nell'esec'uzione delle misure.

(**) Progettate e costruite nell'Istituto di Geofisica Mineraria dell'Universita di Roma, per l'interpretazione di rilievi magnetometrici.

(***) Le dimensioni complessive delle elettrocalamite sono: sezione circa $3 \times 3 \mathrm{~cm}$, lunghezza $3-5-7 \mathrm{~cm}$, il numero di spire, costituite da filo di rame क $0,8 \mathrm{~mm}$, era di 500 per le elettrocalamite di lunghezza $3 \mathrm{~cm}$; di 900 per quelle di lunghezza $5 \mathrm{~cm}$ o di 1200 per quelle di lunghezza $7 \mathrm{~cm}$. 
M. BERNABINI

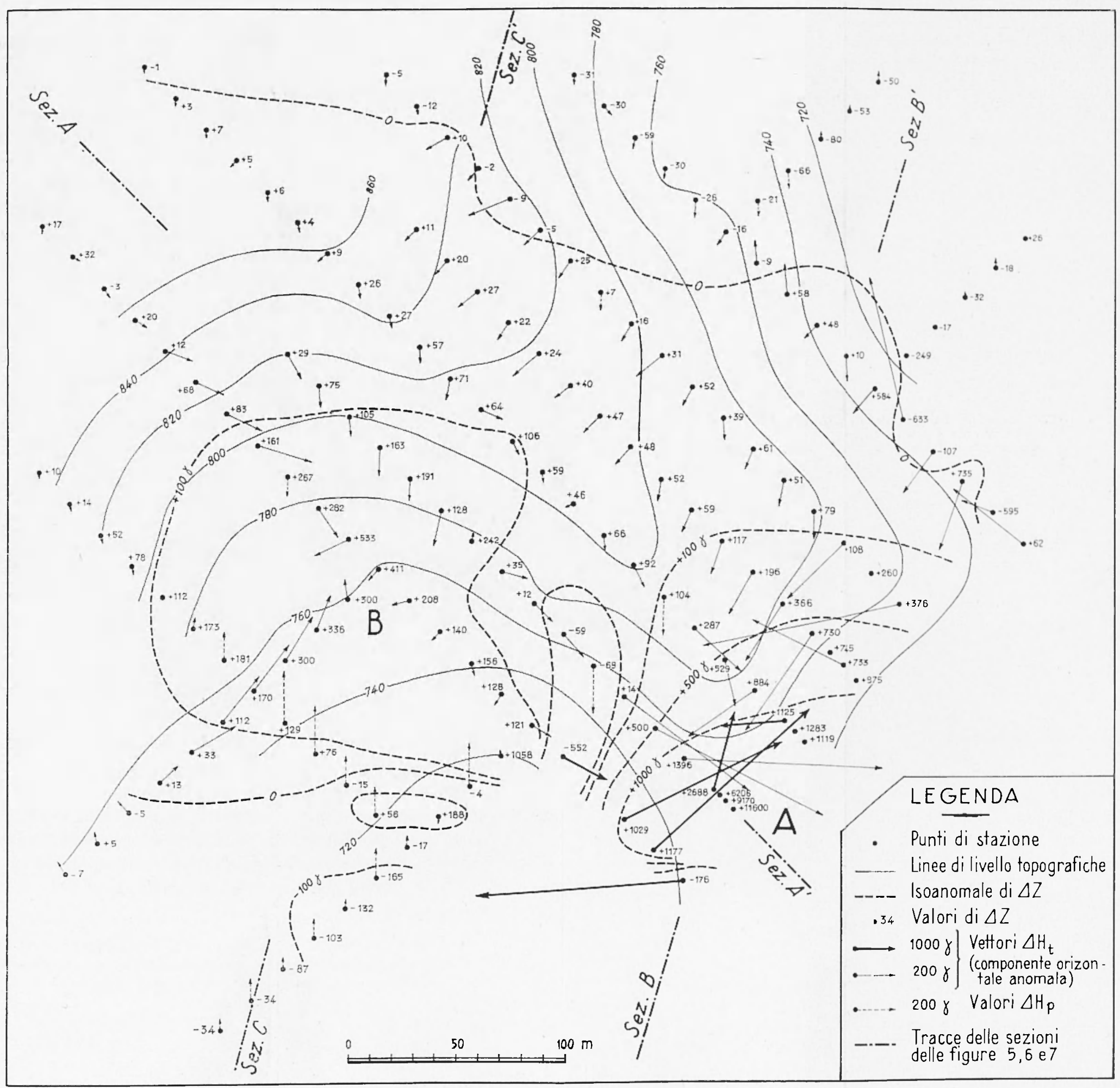

Fig. 3 - Planimetria della zona rilevata. Sono riportati i valori e le isoanomale di $\Delta Z$ e le componenti orizzontali anomale totali $\left(\Delta I_{t}\right)$. 
spire, sia variando la forza elettromotrice di alimentazione, sia con reostati collegati in serie.

Per costruire il modello, si sono montate su di un telaio diverse elettrocalamite, collegate con sistemi misti serie-parallelo, in modo tale che riproducessero in scala approssimata 1:1000, le masse di minerale presunte.

Per giudicare sull'effettiva rispondenza del modello, si è controllato il verificarsi di una similitudine geometrica tra il campo generato dal modello e quello misurato in campagna.

Tale verifica è stata effettuata confrontando l'orientamento delle linee di forza del campo magnetico misurato in campagna nei vari punti di stazione con l'orientamento delle linee di forza del campo generato dalle elettrocalamite nei punti corrispondenti, nel modello, ai punti di stazione.

Praticamente si è operato nel modo seguente:

Si è ruotato il modello in modo da disporre orizzontale la direzione corrispondente, nel modello, alla verticale; si è posta a fianco delle elettrocalamite una lastra di vetro orizzontale, che in tal modo, materializzava, nel modello, uu piano corrispondente ad un piano verticale (Fig. 4 ). Ruotando il modello intorno alla direzione corrispondente alla verticale e traslando la lastra di vetro, si potevano così rappresentare tutti i possibili piani verticali.

Si è sparsa sulla lastra di vetro della limatura di ferro (*); per effetto del campo magnetico generato dalle elettrocalamite, essa, dopo aperto il circuito di alimentazione della corrente, si dispone in modo da rappresentare, sul piano della lastra, la proiezione delle linee di forza del campo (nei punti di intersezione di queste ultime con la lastra).

L'andamento indicato dalla limatura di ferro rappresenta ciò̀ la proiezione del campo magnetico sul generico piano verticale materializzato dalla lastra.

Ta lastra di vetro è stata posta in modo che rappresentasse (nella scala del modello), successivamente tutte le sezioni verticali significative che potevano tracciarsi per i punti di stazione.

E stato effettuato un confronto su ciascuna sezione fra l'andamento del campo indicato nel modello dalla limatura di ferro, e la proiezione del campo magnetico determinato in campagna sulla sezione verticale corrispondente a quella materializzata dalla lastra.

(*) Il quantitativo di limatura da impiegare si è ridotto al minimo possibile per non alterare il campo generato dalle elettrocalamite con la presenza di circuiti magnetici a riluttanza minore di quelli sviluppantisi nell'aria. 
Ciumerosi tentativi sono stati fatti variando il numero, la disposizione, lorientamento e la forza magnetomotrice delle elettrocalamite fino a giungere, per successive approssimazioni, ad una configurazione del modello tale da soddisfare, entro i limiti richiesti, la similitudine geometrica tra il campo del morlello stesso e quello determinato in campagna.

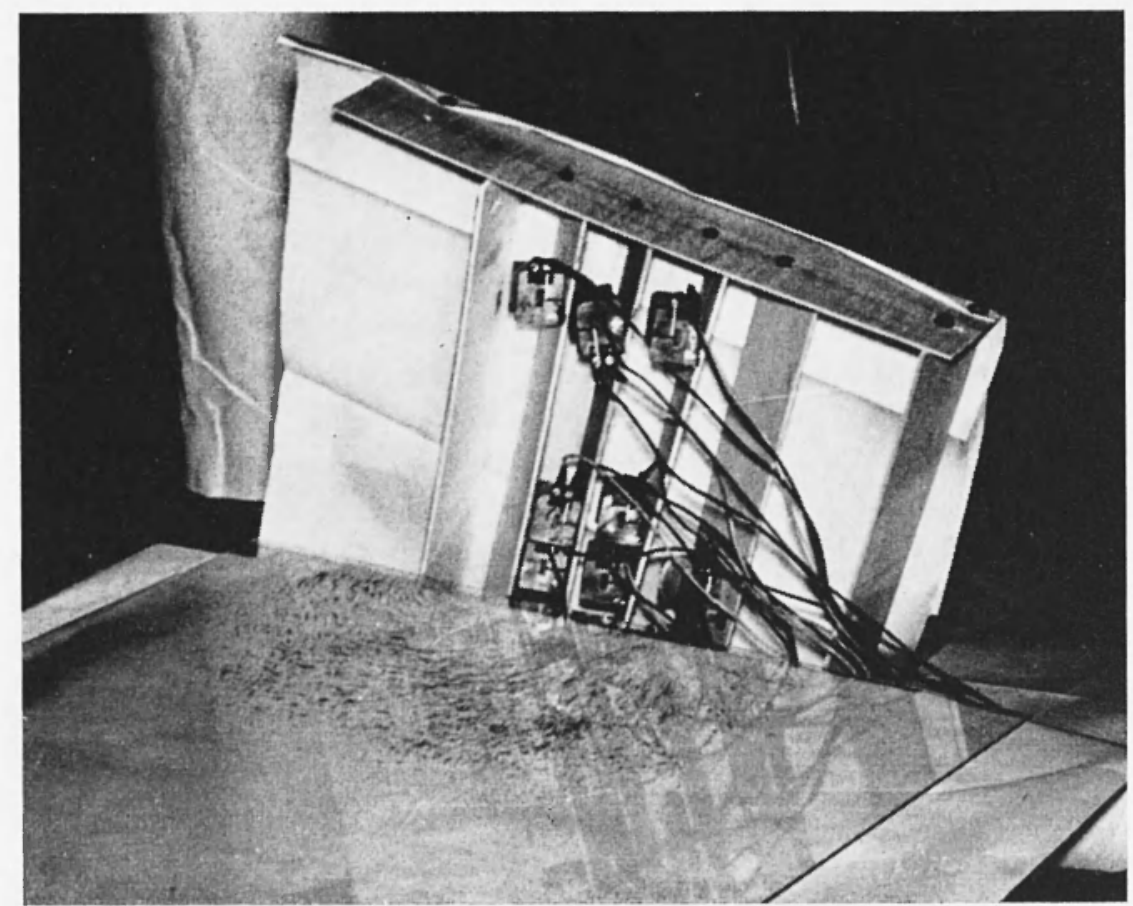

Fir. + Apparecehiatura usata per le prove sui morlelli.

Come esempi nelle Figure 5,6 e 7 sono riportate tre fotografie riguardanti tre sezioni verticali $\left(A-A^{\prime} ; B-B^{\prime} ; C-C^{\prime}\right)$ le cui tracce sono inclicate nella planimetria della Fig. 3. Sulle fotografie sono rappresentati il profilo topoganfico della sezione, la proiezione dei vettori anomali totali e l'andamento delle linee di forza del modello.

Dalla posizione delle elettrocalamite nel modello, è stata ricavata la ubicazione nel sottosuolo delle presumibili masse di minerale magnetico (magnetite).

Inoltre si sono avute indicazioni comparative di prima approssimazione anche sulla grandezza delle varie masse di minerale presente. 
Supponendo infatti che l'intensità di magnetizzazione (indotta $†$ residua) sia costante nell'intero giacimento, i valori dei rapporti tra i momenti magnetici delle singole elettrocalamite, facilmente valutabili conoscendo le caratteristiche delle elettrocalamite e la intensità di corrente passante negli avvolgimenti) dovrebbero corrispondere (in prima approssimazione) ai valori dei rapporti delle masse di minerale di cui le elettrocalamite sono i modelli.

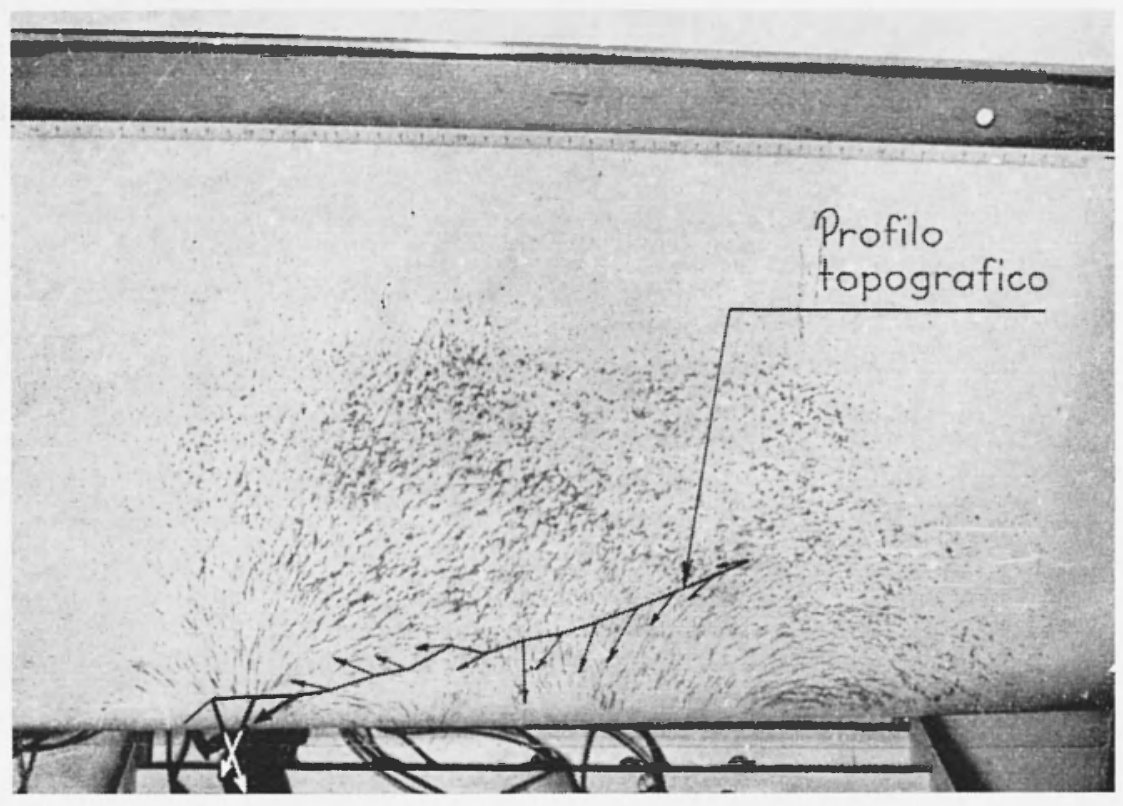

l'ig. 5 - Andamento della proiezione del campo magnetico generato dalle elettrocalamite nel morlello sul piano corrispondente alla sezione verticale $. l-l^{\prime}$ (vedi Fig. 3). Sono anche riportati il profilo topografieo e le proiezioni, sulla sezione, dei vettori anomali totali determinati nelle varie stazioni interessate dalla sezione stessa.

Conoscendo l'entità delle masse mineralizzate nella zona già esplolata con gallerie è stato possibile quindi una valutazione approssimata dell'entità delle masse di minerale di eni, in base ai pisultati del rilievo magnetometrico, si poteva presumere la presenza nelle zone ancora inesplorate.

Con l'interpretazione effettuata ì stato cosi possibile indicane la presenza di due principali complessi mineralizzati, la cui posizione plani- 
metrica è indicata nella Fig. 8, separati da una zona dove la magnetite è assente o molto scarsa.

Il primo complesso $(A)$ corrisponde alla mineralizzazione già esplorata e conosciuta. I risultati del rilievo magnetometrico indicherebbero che tale mineralizzazione non si estende molto oltre le zone interessate dai lavori di ricerca già eseguiti.

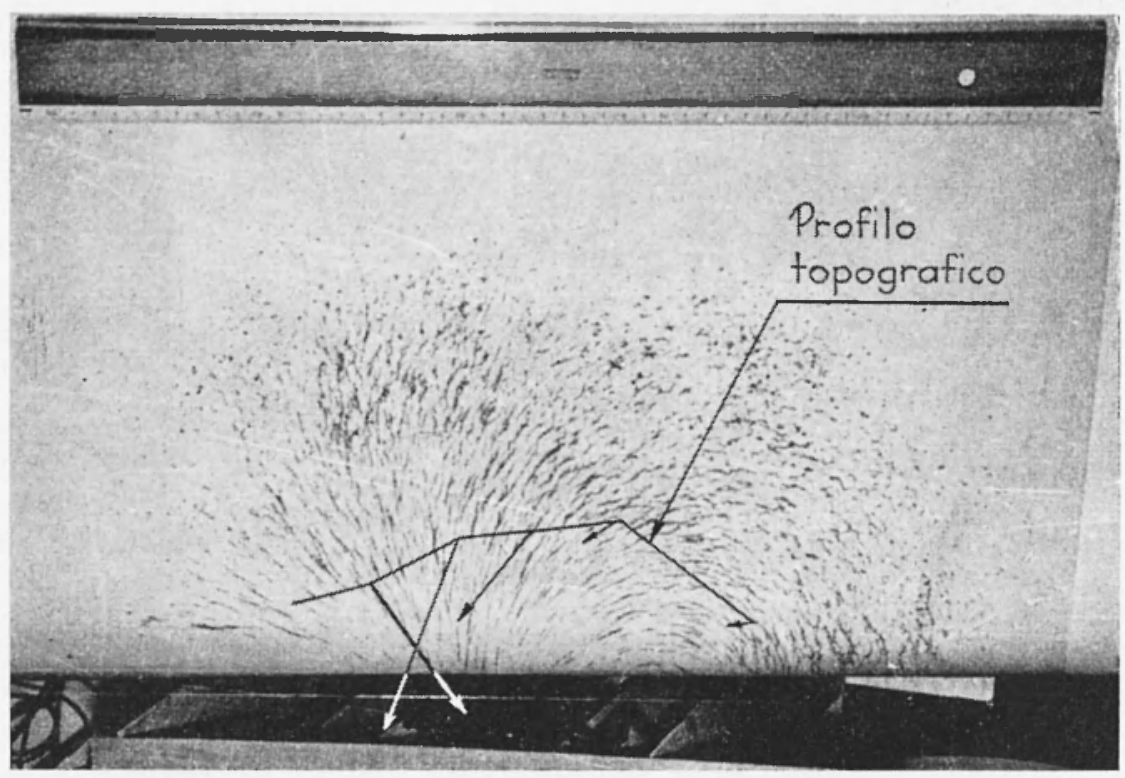

Fig. 6 - Andamento della proiezione del campo magnetico generato dalle elettrocalamite nel modello sul piano corrispondente alla sezione verticale $B-B^{\prime}$ (verli $F i g .3$ ). Sono anche riportati il profilo topografico e le proiezioni, sulla sezione, dei vettori anomali totali determinati nelle varie stazioni interessate dalla sezione stessa.

Nel dettaglio è risultato che il tenore medio in magnetite dovrebbe essere più scar'so nella parte NE del complesso. Ciò è in accordo anche ('on alcune notizie riportate in "Relazioni sul servizio minerario - 1939 " [ $\left.{ }^{5}\right]$ che indicano una prevalenza di pirite nella parte Est della zona interessata dalle ricerche.

Il secondo complesso $(B)$ rlovrebbe essere costituito, schematicamente da una massa magnetica situata a quota eirca $700-720 \mathrm{~m}$ s.l.m. Risulta anche probabile che, nel rettaglio, tale massa sia costituita da varie parti, disposte grosso modo su di un piano, di potenza nella 
verticale in media non superiore a $1 / 2 \div 1 / 3$ di quella delle masse note de] complesso $A$.

Telle zone circostanti non vi dovrebbero essere the poche massc isolate di piccole dimensioni. Le ricerche minerarie effettuate hamno, del resto, individuato solo qualche mineralizzazione a pirite [5].

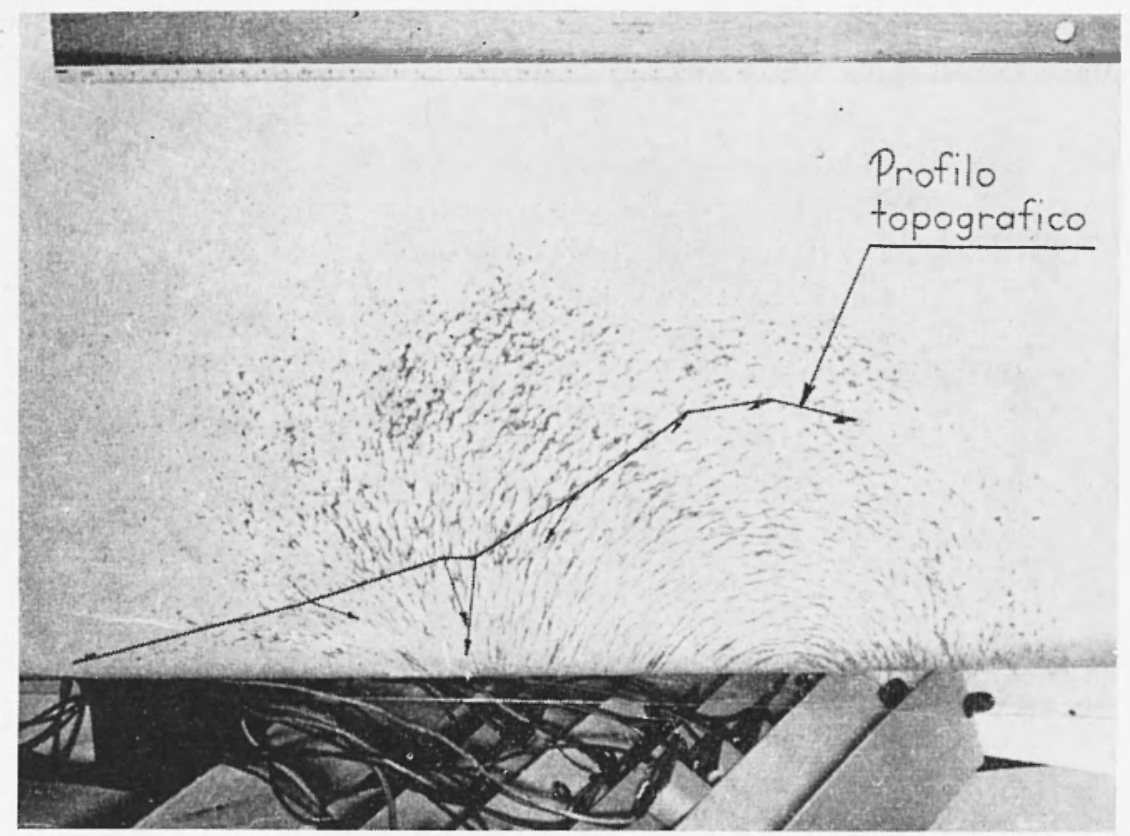

Fig. 7 - Andamento della proiezione del campo magnetico generato dalle elettrocalamite nel modello sul piano, corrispondente alla sezione verticale C. $C^{\prime}$ (vedi Fig. 3). Sono anche riportati il profilo topografico e le proiezioni, sulla sezione, dei vettori anomali totali determinati nelle varie stazioni interessate dalla sezione stessa.

Allo scrivente risulta che, dopo il rilievo magnetometrico, sono stati effettuati lavori di ricerca in galleria verso il complesso $B$ segnalato. Questi lavori sarebbero stati solo di limitatissima entità e non si è potuto pertanto avere ancora un controllo completo della ricostruzione fornita. Sembra tuttavia che in effetti sia stata riconoscinta in corrispondenza della zona indiziata la presenza di magnetite.

Il metodo è stato impiegato anche in un rilievo di dettaglio su un giacimento elbano. In questo caso si è riconosciuto che le anomalie ma- 
gnetiche riscontrate nelle zone di bordo di una massa di magnetite già riconosciuta emano dovute praticamente solo alla massa stessa: questo risultato i stato confermato dai suceessivi lavori minerari.

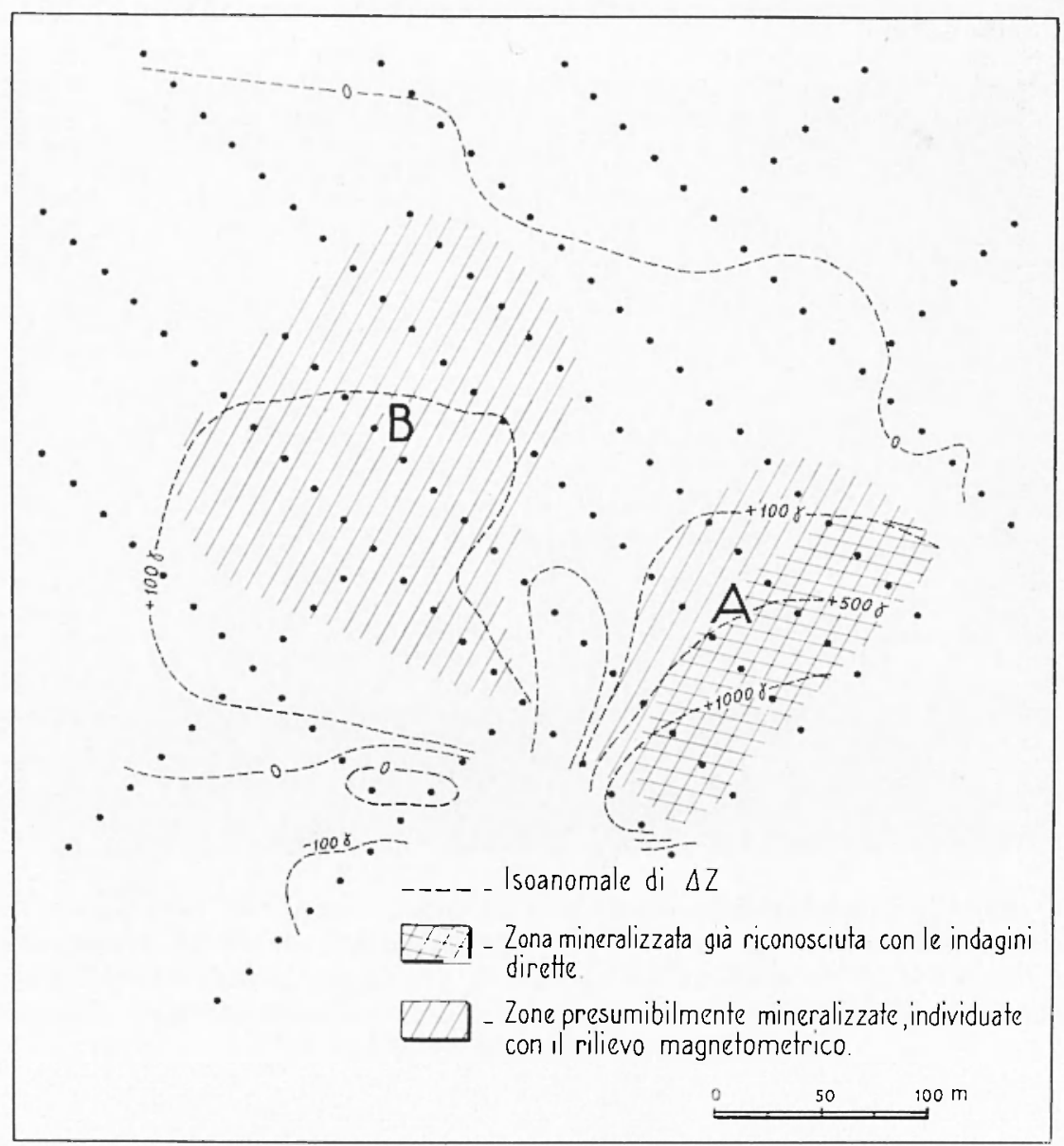

Fig. 8 -- Ubicazione planimetrica delle masse di magnetite individuate con lindagine magnetometriea.

Conchudendo a gemeralizzando i risultati dei rilievi sopra discussi, si può sottolineare l'utilità di effettuare in campagna determinazioni, olte che di $\Delta Z$ a $\triangle H$, anche della declinazione anomala $\Delta D$; l'impiego del vettore anomalo totale, che in tal morlo si può ricavare, rencle infatti 
l'interpretazione più sicura e più spedita soprattutto nei casi di masse irregolari situate in zone a topografia accidentata.

RouA - Luglio 1965

Istituto di Geofisica Mineraria della Facolta d'Ingegneria della Lniversita.

\section{BIBLIOGRAFIA}

(') Aquniva C., Lezioni di Prospezione Geofisica. I, Ed. Ateneo, Roma, 1958.

$\left({ }^{2}\right)$ Narbi R., Geologia della zona tra la Pania della Croce, Gallicano e Castelnuovo Garfagnana (Alpi Apuane). Boll. Soc. Geol. It., LXXX, 2, (1961).

$\left({ }^{3}\right)$ Stelda A. - Manetti L., I giacimenti terriferi delle Alpi Apuane. Le miniere di ferro dell'Italia ». Lattes, Torino, 1921.

(4) Zaccacixa P., Descrizione geologica delle Alpi A puane. Memorie descrittive della carta Geologica d'Italia. XXV. Roma, 1932.

$\left({ }^{5}\right)$ Mrxistero dell'Industria e del Commereio, Rivista del Servizio Minerario e Relazioni sul Servizio minerario. Anni dal 1900 al 1955. 